\title{
Editor's note: Special Issue on Robotics: Science and Systems, 2015
}

Autonomous Robots gratefully acknowledges the editorial work of Dongjun Lee (Seoul National University), Jose Neira (Universidad de Zaragoza) and David Hsu (National University of Singapore) on these five articles for this Special Issue on Robotics: Science and Systems, 2015:

- Robust trajectory optimization under frictional contact with iterative learning. Jingru Luo, Kris Hauser

- Autonomy infused teleoperation with application to brain computer interface controlled manipulation, Katharina Muelling, Arun Venkatraman, Jean-Sebastien Valois, John E. Downey, Jeffrey Weiss, Shervin Javdani, Mar- tial Hebert, Andrew B. Schwartz, Jennifer L. Collinger, J. Andrew Bagnell

- Multipolicy decision-making for autonomous driving via changepoint-based behavior prediction: Theory and experiment, Enric Galceran, Alexander G. Cunningham, Ryan M. Eustice, Edwin Olson

- Guaranteeing spoof-resilient multi-robotnetworks, Stephanie Gil, Swarun Kumar, Mark Mazumder, Dina Katabi, Daniela Rus

- Large-scale, real-time $3 D$ scene reconstruction on a mobile device, Ivan Dryanovski, Matthew Klingensmith, Siddhartha S. Srinivasa, Jizhong Xiao 